\title{
Hydroxyapatite Whiskers Based Resin Composite versus Commercial Dental Composites: Mechanical and Biocompatibility Characterization
}

\author{
L. Calabrese, ${ }^{1}$ F. Fabiano, ${ }^{1,2}$ M. Currò, ${ }^{3}$ C. Borsellino, ${ }^{4}$ L. M. Bonaccorsi, ${ }^{1}$ \\ V. Fabiano, ${ }^{5}$ R. Ientile, ${ }^{3}$ and E. Proverbio ${ }^{1}$ \\ ${ }^{1}$ Department of Electronic Engineering, Chemistry and Industrial Engineering, University of Messina, Contrada di Dio, \\ 98166 Messina, Italy \\ ${ }^{2}$ Department of Experimental, Specialized Medical-Surgical and Odontostomatological Sciences, University of Messina, \\ Via Consolare Valeria 1, 98125 Messina, Italy \\ ${ }^{3}$ Department of Biomedical Sciences and Morpho-Functional Imaging, University of Messina, Via Consolare Valeria 1, \\ 98125 Messina, Italy \\ ${ }^{4}$ Department of Civil Engineering, Computing, Construction, Environmental and Applied Mathematics, \\ University of Messina, Contrada di Dio, 98166 Messina, Italy \\ ${ }^{5}$ Department of Human Pathology, University of Messina, Via Consolare Valeria 1, 98125 Messina, Italy
}

Correspondence should be addressed to L. Calabrese; lcalabrese@unime.it

Received 13 October 2015; Revised 6 January 2016; Accepted 12 January 2016

Academic Editor: Jun Liu

Copyright (C) 2016 L. Calabrese et al. This is an open access article distributed under the Creative Commons Attribution License, which permits unrestricted use, distribution, and reproduction in any medium, provided the original work is properly cited.

A systematic evaluation of mechanical and biocompatibility properties of different volume fractions of hydroxyapatite whiskers in comparison with three commercial dental composites filled with micro- and nanosilica particles was carried out. Six groups with different hydroxyapatite whiskers mass fractions were taken into account in order to be compared with the performances of silica particles based composites group. Flexural properties were evaluated via a universal testing machine (2.5 kN Zwick Line) with a $2 \mathrm{kN}$ load-cell (sensitivity $0.001 \mathrm{~N}$ ). The test was replicated 10 times for the seven experimental groups to better identify statically the significance of the mechanical performances data. MTT quantitative colorimetric assay was performed in order to evaluate the mitochondrial activity of living cells exposed to different resin composites. Data obtained show better interfacial interaction with filler/matrix until $20 \mathrm{wt} \%$ of hydroxyapatite whiskers partially replaced silica particles filler. After this threshold, the mechanical performances decrease dramatically due to both the hydroxyapatite agglomerates formation and the low degree of resin conversion. In addition, biocompatibility test showed less cytotoxic effect with the addition of $20 \mathrm{wt} \%$ of hydroxyapatite in comparison with higher rates.

\section{Introduction}

Nowadays, in order to avoid limits affecting long-term durability of resin composites, dental materials companies are focusing their attention on the improvement of the mechanical and biological properties of filler. Thus, working on filler packing, optimization of filler content, and development of a new innovative fillers is needed.

Hydroxyapatite (HA), $\mathrm{Ca}_{10}\left(\mathrm{PO}_{4}\right)_{6}(\mathrm{OH})_{2}$, was introduced since 1975 as filling material for intrabony defects [1-4].
As the main biomineral component in enamel and dentine, hydroxyapatite is responsible for their higher mechanical performances. In fact, enamel shows an average of about $90 \mathrm{GPa}$ for the elastic modulus and $4.80 \mathrm{GPa}$ for hardness, while dentin shows an average of $0.90 \mathrm{GPa}$ for hardness and $20 \mathrm{GPa}$ for the elastic modulus [5].

For this reason, in the recent five years hydroxyapatite particles and whiskers have been added as novel bioactive and biocompatible reinforcing filler in dental restorations. In fact, it was shown that the incorporation of hydroxyapatite 
with whiskers morphology can provide larger load transfer and favor toughening mechanisms, thus, increasing flexural modulus and fracture toughness of resins in comparison with conventional silica based fillers [6-11]. In addition, hydroxyapatite whiskers (wHA) have been considered the best option as reinforcing filler in comparison with other whiskers based on carbon, ceramic, glass, metal, and polymer due to the absence of cytotoxicity effects $[12,13]$.

Due to its good cation exchange rate with metals, $\mathrm{Ca}_{10}\left(\mathrm{PO}_{4}\right)_{6}(\mathrm{OH})_{2}$ represents the most promising substrate for potential release of antimicrobial molecule or ions (i.e., silver, copper, and zinc). This approach is promising in order to prevent the major bacterial biofilm retention of resin composite which is the main reason of secondary caries insurgence and then restoration failure [14].

To overcome the present lack in the literature about hydroxyapatite based composite, we proposed a systematic analysis of the mechanical and biocompatibility performances of them in comparison with commercial dental composite. For wHA filled composite resins, several efforts are still necessary to enhance the interphase combination and investigate the overall properties [8].

This information is of primary interest in order to achieve a final consistency and specific formulation of hydroxyapatite whiskers mixed with silica particles filler in resin composite. In particular, six different ratios of hydroxyapatite/silica particles were investigated.

With this aim, different mass fraction of hydroxyapatite based composites and three commercial composites loaded with silica particles were tested and compared in order to verify the effective enhancement in both biocompatibility and mechanical properties. In particular, three-point bending test, Vickers hardness, morphological analysis, and quantitative colorimetric assay to assess cell viability were performed.

\section{Materials and Methods}

2.1. Materials. Camphorquinone (CQ), ethyl 4-dimethylaminobenzoate (EDMAB), 2,2-bis[p-( $2^{\prime}$-hydroxy- $3^{\prime}$ methacryloxypropoxy)phenyl]propane (Bis-GMA), triethylene glycol dimethacrylate (TEGDMA), calcium nitrate tetrahydrate $\left(\mathrm{Ca}\left(\mathrm{NO}_{3}\right)_{2} \cdot 4 \mathrm{H}_{2} \mathrm{O}\right)$, ammonium phosphate dibasic $\left(\left(\mathrm{NH}_{4}\right)_{2} \mathrm{HPO}_{4}\right)$, and urea $\left(\left(\mathrm{NH}_{2}\right)_{2} \mathrm{CO}\right)$ were purchased from Sigma-Aldrich Company (Milano, Italy). The inorganic filler based on $0.7 \mu \mathrm{m}$ size silica particles was provided by Esstech Inc. (Essington, USA).

The human premonocytic cell line, THP-1, was obtained from DSMZ (Braunschweig, Germany). RPMI-1640 medium, penicillin/streptomycin mixture, L-glutamine, 4(2-hydroxyethyl)-1-piperazineethanesulfonic acid (HEPES), sodium pyruvate, glucose, fetal bovine serum (FBS), 3(4,5-methylthiazol-2-yl)-2,5-diphenyl-tetrazolium bromide (MTT), and other chemicals of analytical grade were purchased from Sigma Aldrich (Milano, Italy).

2.2. Synthesis of HAP Whiskers. Hydroxyapatite whiskers (wHA) were prepared by hydrothermal homogeneous precipitation at $180^{\circ} \mathrm{C}$ (in autogenous pressure) for $10 \mathrm{~h}$. An aqueous solution containing stoichiometric amounts of

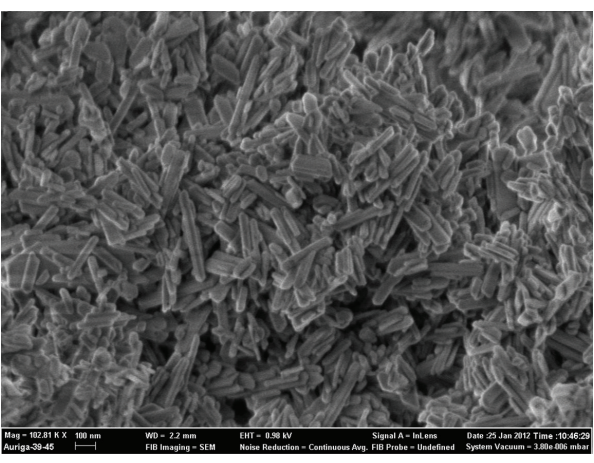

FIGURE 1: SEM image of synthesized whiskers.

$\mathrm{Ca}\left(\mathrm{NO}_{3}\right)_{2} \cdot 4 \mathrm{H}_{2} \mathrm{O}\left(10-334 \mathrm{mmol} \mathrm{L}^{-1}\right)$ and $\left(\mathrm{NH}_{4}\right)_{2} \mathrm{HPO}_{4}$ (6$200 \mathrm{mmol} \mathrm{L}^{-1}$ ) with a final molar ratio $\mathrm{Ca} / \mathrm{P}$ of 1.67 was prepared. The solution $\mathrm{pH}$ was adjusted to 3 by adding urea $\left(\mathrm{CO}\left(\mathrm{NH}_{2}\right)_{2}\right)$. The resulting mixture was then poured into a PTFE container inside a stainless steel autoclave and put in an oven at $180^{\circ} \mathrm{C}$ for 10 hours. The final solution was centrifuged to recover the precipitate powder, which was washed with distilled water and dried at $80^{\circ} \mathrm{C}$ overnight.

Figure 1 shows SEM micrograph of synthesized HA whiskers. Whiskers are well defined and mainly monodisperse, although some ones that fused each other can be identified.

The aspect ratios (i.e., length/diameter) varied in the range $2 \pm 10$, with average whisker diameter and length $35 \mathrm{~nm}$ and $200 \mathrm{~nm}$, respectively.

2.3. Dental Composite Preparation. Dental resin matrix was obtained mixing $49.5 \mathrm{wt} \%$ Bis-GMA and $49.5 \mathrm{wt} \%$ diluent comonomer TEGDMA. Camphorquinone and ethyl 4dimethylaminobenzoate were added as the initiator and coinitiator in the ratio of $0.5 \mathrm{wt} \%$, respectively. Various volume fractions of HA whiskers were added into organic matrix replacing the silica particles, in particular from 0 vol\% to 100 vol\% HA whiskers as reported in Table 1.

Furthermore, three commercial resin composites were chosen as mechanical and biocompatibility evaluation reference in this work:

(i) Quadrant universal LC is a light-cured, fluoride releasing radiopaque microglass composite for universal application. It is based on a Bis-GMA matrix and contains $60 \%$ filler by volume or $72 \%$ by weight, which is formed by Ba-Al-F-silicate glass $(0.02-2 \mu \mathrm{m})$ and highly dispersed silicon dioxide $(0.02-0.07 \mu \mathrm{m})$.

(ii) Durafill is a microfilled light-cured composite filling material with a total amount of $66 \%$ of inorganic and organic fillers by volume. The filler contains highly disperse silicon dioxide $(0.02-0.07 \mu \mathrm{m})$ and splinter polymer $(<20 \mu \mathrm{m})$.

(iii) Renamel microfill is a microfilled light-cured composite filling material which consists of multifunctional acrylic resins and fillers of 0.04-0.2 micron sized particles of inorganic and prepolymerized composite ( $70 \%$ by weight and $60 \%$ by volume). 
TABLE 1: Different volume fraction of HA whiskers and inorganic filler in resin based composite formulation.

\begin{tabular}{lcccccc}
\hline CODE & HA density $\mathrm{g} \backslash \mathrm{cm}^{3}$ & Silica density $\mathrm{g} \backslash \mathrm{cm}^{3}$ & \% Vol HA (\%wt) & \% Vol Silica (\%wt) & HA $\backslash$ Silica Vol ratio & \% Vol resin \\
\hline HA-0 & 3.1 & 2.4 & $0.0 \%(0.0 \%)$ & $30.0 \%(46.2 \%)$ & $0.0 \%$ & $30.0 \%$ \\
HA-20 & 3.1 & 2.4 & $6.0 \%(11.6 \%)$ & $24.0 \%(36 \%)$ & $20.0 \%$ & $30.0 \%$ \\
HA-40 & 3.1 & 2.4 & $12.0 \%(22.6 \%)$ & $18.0 \%(26.3 \%)$ & $40.0 \%$ & $30.0 \%$ \\
HA-60 & 3.1 & 2.4 & $18.0 \%(33.1 \%)$ & $12.0 \%(17.1 \%)$ & $60.0 \%$ & $30.0 \%$ \\
HA-80 & 3.1 & 2.4 & $24.0 \%(43.1 \%)$ & $6.0 \%(8.3 \%)$ & $80.0 \%$ & $30.0 \%$ \\
HA-100 & 3.1 & 2.4 & $30.0 \%(52.5 \%)$ & $0.0 \%(0.0 \%)$ & $100.0 \%$ & $70.0 \%$ \\
\hline
\end{tabular}

2.4. Mechanical Test. Flexural properties were evaluated using rectangular-shaped specimens with dimensions: $25 \mathrm{~mm} \times 2 \mathrm{~mm} \times 2 \mathrm{~mm}$. To make the samples, a customized stainless steel mold was used where the lateral sides are made by two glass slides with a polyester film interposed between the glass and the mold. Such mold was filled with the uncured resin composite, then the glass slide was removed, and the exit window of a visible light unit (Optilux-501, Kerr, CT, USA) with a wavelength of 400-505 $\mathrm{nm}$ and output of $1000 \mathrm{~mW} / \mathrm{cm}^{2}$ was positioned at the center of the specimen, against the glass slide, so that the specimen was irradiated for polymerization time of $60 \mathrm{~s}$. After the photoactivation of the specimen's center, the exit window was moved to the section next to the center, overlapping the previous section by half the diameter of the exit window, the same for the section on the other side of the center. After that, both sides were irradiated, and the specimens were stored in distilled water at $37^{\circ} \mathrm{C}$ for $24 \mathrm{~h}$ before testing, according to ISO 4049 standard.

Three-point bending test was performed at room temperature using a universal testing machine (2.5 kN Zwick Line) $2 \mathrm{kN}$ load-cell (sensitivity $0.001 \mathrm{~N}$ ). The cross-head speed was $0.75 \mathrm{~mm} / \mathrm{min}$.

Microhardness tests were performed by using a FutureTech Microhardness Tester FM-300 (Vickers indenter and compressive load $100 \mathrm{~g}$ ). Surface microstructure of hydroxyapatite whiskers and resin composite was examined by focused ion beam scanning electron microscopy (FIB-SEM Crossbeam Zeiss).

2.5. Cell Culture and Viability Test. THP-1 cells were maintained in RPMI 1640 supplemented with L-glutamine $(2 \mathrm{mM})$, HEPES $(10 \mathrm{mM})$, sodium pyruvate $(1 \mathrm{mM})$, glucose (2.5 g/l), 2-mercaptoethanol (0,05 mM), 10\% heat-inactivated fetal bovine serum (FBS), and 1\% penicillin/streptomycin, at $37^{\circ} \mathrm{C}$ in a $5 \% \mathrm{CO}_{2} / 95 \%$ air humidified atmosphere. Medium was renewed every 2 days and split performed when cells reached maximum density $\left(1 \times 10^{6}\right.$ cells $\left./ \mathrm{ml}\right)$. In our experimental conditions, THP-1 cells were seeded at a density of 5 $\times 10^{5}$ cells $/ \mathrm{ml}$ into culture plates in RPMI complete medium plus $10 \% \mathrm{FBS}$ and incubated at $37^{\circ} \mathrm{C}$ for $48 \mathrm{~h}$ with various composite powders, containing different rate of HA whiskers, at concentrations ranging between 0.05 and $1 \mathrm{mg} / \mathrm{ml}$.

To assess the effects of different composites on cell viability, we evaluated the mitochondrial activity of living cells by a MTT quantitative colorimetric assay. After treatment, THP-1 cells were harvested by centrifugation and incubated in 96-well culture plates with fresh red-phenolfree medium containing MTT $(0.5 \mathrm{mg} / \mathrm{mL})$ at $37^{\circ} \mathrm{C}$ for $4 \mathrm{hs}$. Then, insoluble formazan crystals were dissolved in $100 \mu \mathrm{L}$ of a $0.04 \mathrm{~N} \mathrm{HCl} /$ isopropanol solution for $1 \mathrm{~h}$. The optical density in each well was determined at $570 \mathrm{~nm}$ using a microplate reader (Tecan Italia, Cologno Monzese, Italy).

2.6. Statistical Analysis. Results were expressed as mean \pm standard error of the mean for each group of specimens. One-way analysis of variance (ANOVA) tests were performed for the dependent variables such as elastic modulus, flexural strength, and cell viability. In particular, flexural tests were performed in 10 replicas for each group, while biocompatibility test was replicated five times for each experimental group. Student-Newman-Keuls post hoc test was used for multiple comparison and statistical significance was considered at $p<$ 0.05 .

\section{Results and Discussion}

In Figure 2, three-point flexural test for a HA-20 resin composite samples is shown as reference because of showing the better mechanical performances between HA-based experimental groups. Stress (on the main axis) and modulus (on the secondary axis) are plotted at increasing the strain.

Analyzing the figure, three significant regions can be identified.

At first, the initial trend of stress-strain curve is related with the mechanical adjustment phases of the set-up configuration. This section of the curve is usually quite disturbed and it is not indicative of the mechanical behavior of the sample.

After the stabilization phase, the stress increases quite linearly with the strain. In this phase, the relation between stress and strain can be considered linear (Figure 2) and the modulus, $E$, is quite constant $\left(E_{\max }\right.$ about $\left.3500 \mathrm{MPa}\right)$. In this phase, the material has a pure linear elastic behavior with limited strain values (up to 0.003 ).

On the contrary, at higher deformations, the relationship between stress and strain becomes nonlinear and the stiffness progressively decreases. This phase is influenced by the viscous behavior of the matrix. In this region, the interfacial filler-matrix strength plays an important role on the mechanical performance of the composite paste, influencing trend slope and threshold interfacial stresses.

Finally, a mainly brittle fracture occurred at 0.03 strain. The maximum stress, observed at failure was about $55 \mathrm{MPa}$. 


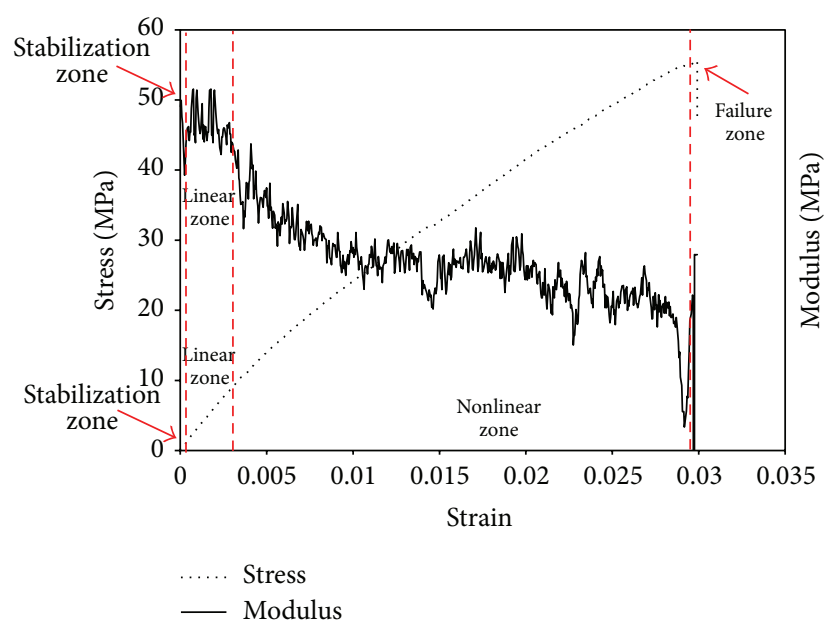

FIgURE 2: Young's modulus (continuous line) and flexural strength (dotted line) in function of deformation for a HA-20 reference composite sample.

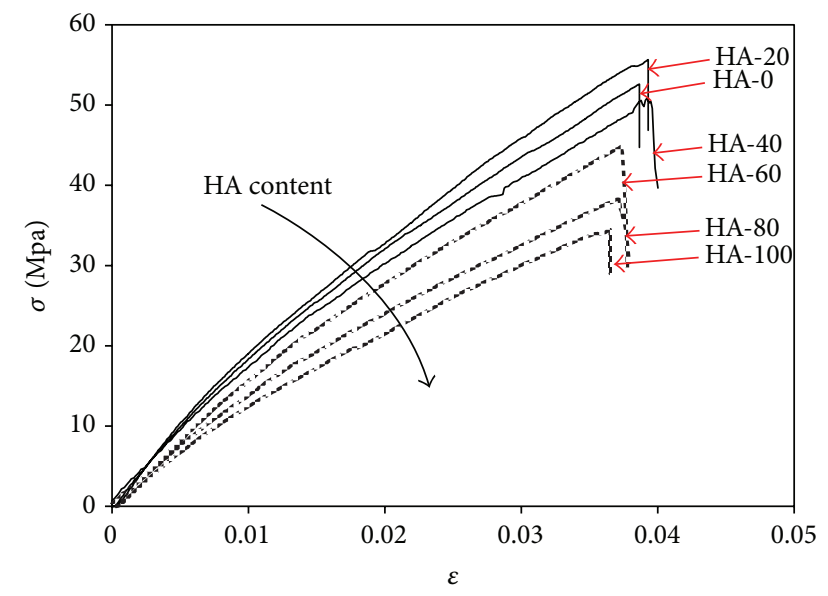

Figure 3: Stress-strain curves of samples at different mass fraction of hydroxyapatite whiskers filled resin composite.

In order to evaluate the effect on the mechanical performances of the hydroxyapatite addiction in the filler mixing composition of the composite paste, the stress-strain curve evolution at increasing HA amount is shown in Figure 3. The mechanical performances of the composite material are influenced by the addiction of HA whiskers. At first, a slight increase of failure stress can be observed for HA-20 sample. Afterwards, a progressive reduction of maximum stress and modulus (slope of stress-strain curve) can be identified at increasing $\mathrm{HA}$ amount (with the consequent reduction of silica filler content). Furthermore, the strain failure progressively decreases at increasing HA amount. Samples with a large amount of hydroxyapatite evidenced a significant reduction of mechanical performances.

Analyzing in detail stress-strain curves reported in Figure 3,0\% HA/Silica and 20\% HA/Silica samples are able to carry high stresses and strains combining high stiffness and strength. In fact, they can reach high deformation without the insurgence of critical failure conditions. These
TABLE 2: Vickers hardness of different mass fraction of hydroxyapatite based composites.

\begin{tabular}{lc}
\hline Samples & Vickers hardness $(\mathrm{HV})^{\mathrm{a}}$ \\
\hline HA-0\% & $58(9)$ \\
HA-20\% & $77(6)$ \\
HA-40\% & $69(12)$ \\
HA-60\% & $57(7)$ \\
HA-80\% & $55(10)$ \\
HA-100\% & $53(8)$ \\
\hline
\end{tabular}

${ }^{\mathrm{a}}$ Mean value (standard deviation).

samples evidenced a slight nonlinear curve with a first elastic region until medium deformation (about 0.005). Afterwards, at increasing deflections the stress-strain curve exhibits a progressive moderate deviation from linear trend. Anyway a mainly linear elastic behavior can be observed for these samples (HA-0 and HA-20). These samples maintain an elastic regime also at high strain, evidencing an elasticbrittle behavior typical for well cross-linked composite resin $[15,16]$. Thus, the interfacial interaction between filler and matrix, which participate positively in stress transfer between composite constituents, becomes the driving force in the strengthening and toughening effects on the particular composite [17].

At increasing hydroxyapatite content, a progressive increase of viscous contribution in the mechanical behavior of the composite paste can be observed. HA- 80 and HA-100 samples exhibited an elastoplastic behavior, as confirmed by the nonlinear trend of the stress-strain curve. The low performances observed for large amount of HA could be due to a reduction of cohesive strength at the matrix-filler interphase that favors low failure stressed. At the same time, the hydroxyapatite induces a shielding effect on the photoactivation of the resin on the bulk [18]; consequently, a large amount of HA filler could act as inhibitor of the photoactivation of the resin inducing nonoptimal crosslinking [19]. This is confirmed by the Vickers hardness data reported in Table 2. The degree of conversion is defined as the percentage of reacted $\mathrm{C}=\mathrm{C}$ double bonds [20]. Hardness has been shown to be a good indicator of conversion of double bonds and was therefore used in the present study as indirect measurements of conversion. The unreacted compounds, characterized by high mobility, influence significantly the plastic deformation inducing low strength and stiffness on the sample. In fact, if the deepest layers of composite restorations are not adequately cured, the elastic modulus at the bottom will be lower than that at the surface; this can increase the material strain under masticatory forces [21]. At the same time, nonhomogenous reticulation of the composite paste stimulates differential polymerization shrinkage stresses inducing tensile stresses on the core of resin composite. Furthermore, the premature failure at both low stress and strain values could indicate a reduced cohesive strength of the dental restorative paste due to a nonoptimal interfacial interaction of the composite fillers with the matrix.

The variation of flexural strength against wHA filler loading is shown in Figure 4. Each datum in the plots provides 
TABLE 3: Statistically significant differences on flexural strength values were evaluated by one-way ANOVA, $P<0.05$.

\begin{tabular}{lcccccr}
\hline Source & SS & df & MS & $F$ & $\begin{array}{c}\alpha \\
F_{\text {critical }}\end{array}$ & $\begin{array}{c}5 \% \\
P \text { value }\end{array}$ \\
\hline Between & 6209.998 & 5 & 1242.000 & 61.322 & 2.386 & 0.000 \\
Within (error) & 1093.703 & 54 & 20.254 & & & Reject \\
Total & $\mathbf{7 3 0 3 . 7 0 1}$ & $\mathbf{5 9}$ & & & & \\
\hline
\end{tabular}

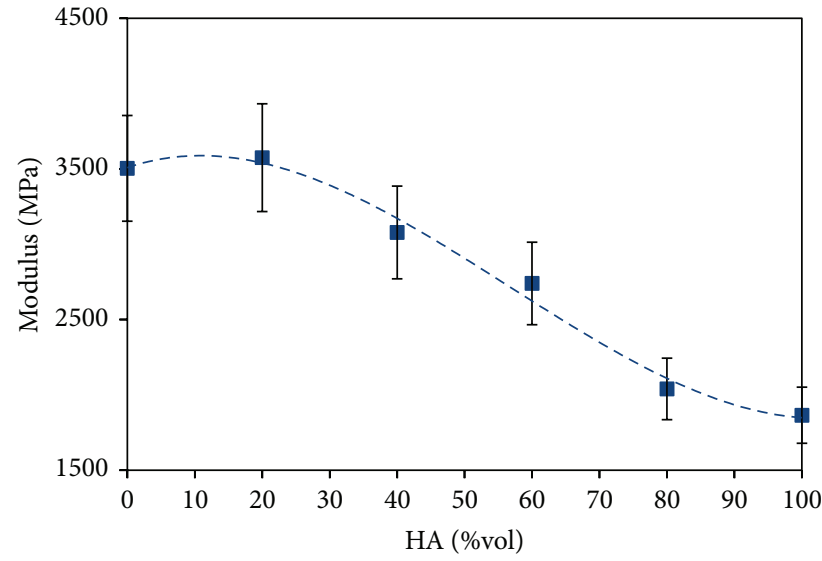

FIGURE 4: Failure strength and Young's modulus values at increasing HA/Silica filler ratio. Errors bars are standard deviation.

the mean value of 10 measurements with standard deviation reported as $y$ error bar. Mechanical performances decreased increasing wHA volume fraction. In fact, at low amount of hydroxyapatite whiskers (until $20 \mathrm{wt} \%$ of HA), the flexural strength of the composite paste is quite constant, maintaining $s$ stable maximum stress and modulus of about, respectively, $55 \mathrm{MPa}$ and $3500 \mathrm{MPa}$.

The mechanical performances progressively decrease at increasing $\mathrm{HA}$ amount. The $60 \% \mathrm{HA} /$ Silica samples show a reduction of about $24.1 \%$ of Young modulus and $21.8 \%$ of maximum stress compared to $0 \% \mathrm{HA} / \mathrm{Silica}$ samples. This behavior could be explained considering that the composite reinforced with HA is characterized by a reduced cohesive strength with higher heterogeneity than the commercial composite which favors a premature fracture at lower stress level. Finally, above $70 \%$ of HA, the mechanical properties of the composite materials are very low. A further increase of HA amount does not induce significant variation of flexural strength, observing a plateau at about $32 \mathrm{MPa}$ of stress and $1950 \mathrm{MPa}$ of Young modulus. These low mechanical performances can be related to the untreatment with silane of hydroxyapatite fillers, as reported also by Santos et al. [9]; that is less relevant until $20 \mathrm{wt} \%$ of HA which permits a better contact with the polymer matrix.

The results summarized in Figure 4 evidence that affordable mechanical performances can be obtained with the addiction of reduced content of HA filler (under 40\%) evidencing an effective interaction between silica filler, hydroxyapatite whiskers, and Bis-GMA based matrix. The percentage of HA of $40 \%$ can be considered a threshold value above witch the mechanical properties are not suitable for restoration applications. In fact the Young modulus at $20 \mathrm{wt} \%$ of wHA was $3497.82 \mathrm{MPa}$ while at $0 \mathrm{wt} \%$ was $3560.65 \mathrm{MPa}$ with a nonsignificant decrease of $1.7 \%$. Instead, the difference between $20 \mathrm{wt} \%$ and $80 \mathrm{wt} \%$ of wHA filler highlights a further decrease of $43 \%$ despite the composite resin with $20 \mathrm{wt} \%$ of HA. The Young modulus for HA-100 points out a decrease of about $50 \%$ in comparison with data obtained with the resin composite filled with the $20 \mathrm{wt} \%$ of wHA, confirming that with large amount of $\mathrm{HA}$ particles the mechanical performances are compromised.

However, the addition of filler to the matrix may increase the adhesive interaction to a tooth substrate [22] and decrease the degradation of the material over time [23], without prejudicing its mechanical performances. The group with $20 \%$ HA showed very affordable mechanical performances with no significant difference compared to the control group, HA0 (a slight enhancement of average mechanical performances can be observed, although the spread data indicate that differences are not statistically significant). Consequently, according to [21], the addition of low amount of HA filler to a commercial hybrid layer could maintain stable mechanical properties of this layer and increase the bond strength. Instead, samples with higher amounts of HA filler evidenced low mechanical properties with subsequent lower bond strength when applied for restorative dental applications.

The influence of the HA content factor on modulus and failure stress of the composite resin has been evaluated by ANOVA (for $P=0.05$ ) and performed by Minitab ${ }^{\circledR}$ software. The results are summarized in Tables 3 and 4 .

Considering that from the ANOVA the $P$ value is zero $(P=0.000<0.05)$ for both analyses, then it is unlikely that the differences observed are due to random sampling.

This rejects the null hypothesis and concludes that not all of population means are equal. Consequently, the hypothesis that the populations of samples at varying HA content have statistically identical means can be rejected. This implies that modulus and stress are influenced by HA addiction on the filler composition, and consequently "HA content" is statistically significant factor for the mechanical performances of composite resin.

Interesting consideration can be extrapolated better analyzing the filler-matrix interaction for $80 \% \mathrm{HA} /$ Silica samples as shown in the SEM micrograph reported in Figure 5. This analysis allows us to evaluate the morphology of the composite material and to study its fracture mechanics. The structure of the HA-80 composite paste consists of a central core of Bis-GMA based resin surrounded by whiskers of HA having micrometric dimensions (length $\approx 4-5 \mu \mathrm{m}$ ).

This morphology is due to nonoptimal mixing between the resin and the filler, due to the high viscosity induced by 
TABLE 4: Statistically significant differences on flexural modulus values were evaluated by one-way ANOVA, $P<0.05$.

\begin{tabular}{lcccccr}
\hline Source & SS & df & MS & $F$ & \multicolumn{2}{c}{$\begin{array}{c}5 \% \\
F_{\text {critical }}\end{array}$} \\
\hline Between & 26293419.20 & 5 & 5258683.840 & 71.937 & 2.386 & 0.000 \\
Within (error) & 3947483.20 & 54 & 73101.541 & & & Reject \\
Total & $\mathbf{3 0 2 4 0 9 0 2 . 4 0}$ & $\mathbf{5 9}$ & & & & \\
\hline
\end{tabular}

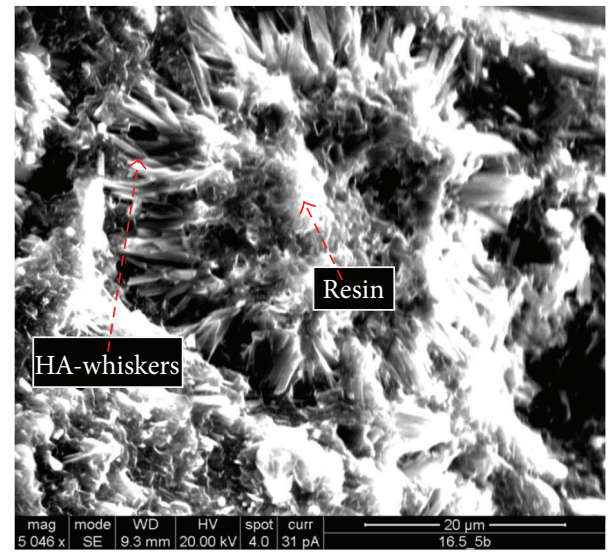

FIGURE 5: SEM micrograph at high magnification of the composite resin reinforced with hydroxyapatite whiskers.

the addition of large amount of hydroxyapatite [21]. Since the volume percentage of filler of the composite is high, the resin is not sufficient to wet the fillers and microdomains consisting of resin particles coated ceramic filler are created.

As in all composite systems, filler reinforcement efficacy depends substantially on the quality and typology of the interface via matrix links.

In order to increase the threshold filler content, Zhang and Darvell [8] explored the effect of silanization of wHA and filler loading on properties of the composite resin and compared the results with hydroxyapatite particle filler, demonstrating the better reinforcing ability and bioactivity of whisker.

However, the influence of silanization was not deeply investigated, and voids between whiskers and matrix lead to undesirable low adhesion strength favoring premature failure at low stress level.

The morphology seen in Figure 5 may be represented as a micelle structure (Figure 6). The core is made of BisGMA resin surrounded by the needle-like HA filler arranged radially in space with respect to the nucleus, assuming a shape similar to a hedgehog. The whiskers are not distributed homogeneously around the core of the resin, but they are anchored on the surface assuming random orientations and distributing the filler not uniformly across the surface of the core. Consequently the micelle is characterized by a combination of dense and voids areas.

The nonoptimal interaction between the filler and the matrix implies that regions with hydroxyapatite not impregnated with resin are created; in particular that occurs when the HA whiskers are aggregated and the resin cannot

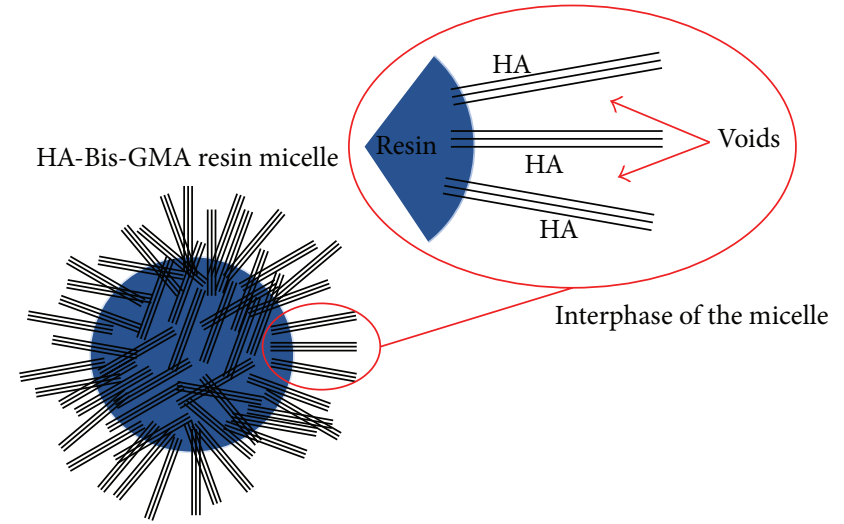

FIGURE 6: Schematization of hydroxyapatite whiskers/matrix interaction. Three fundamental components can be highlighted in the composite: the resin which consists predominantly of Bis-GMA which is shown in blue, the filler of hydroxyapatite (HA) with needle-like morphology (whiskers) and micrometric size, and the voids in white.

penetrate into the interstices. Hydroxyapatite is anchored to the resin core only in correspondence of a small portion, but almost all of its length is not wet; the radial arrangement of the whiskers of HA hinders the permeation of resin in the spaces between the various lamellae which then remains empty (magnification shown in Figure 5); consequently the voids formation is favored.

Based on these considerations, deduced analyzing Figure 5 , it is possible to schematize a three-dimensional structure of the HA-Bis-GMA composite (Figure 6): it can be seen as a set of micelles having a shape like a hedgehog arranged in a disordered manner in a bulk of Bis-GMA resin.

The amount of voids is remarkable because each micelle has an intrinsic amount of dry regions related to poor wetting and compatibility between the HA filler and the polymeric Bis-GMA matrix.

Within the framework of the HA-Bis-GMA composite (Figure 7), we can distinguish three different zones. In zone 1 , the presence of voids (air bubbles) is observed. They could act as defects inducing an increase of stress-intensity factor. In 2 and 3 zones, the rigid HA whiskers of the micelles are in contact with each other favoring the transfer of the stresses that lead to a stiffening of the composite. This could explain the slight increase of elastic modulus $\mathrm{E}$ of the composite reinforced with lower amount of HA compared to commercial composite. Instead, for high amount of HA whiskers, the large presence of voids due to nonoptimal filler 

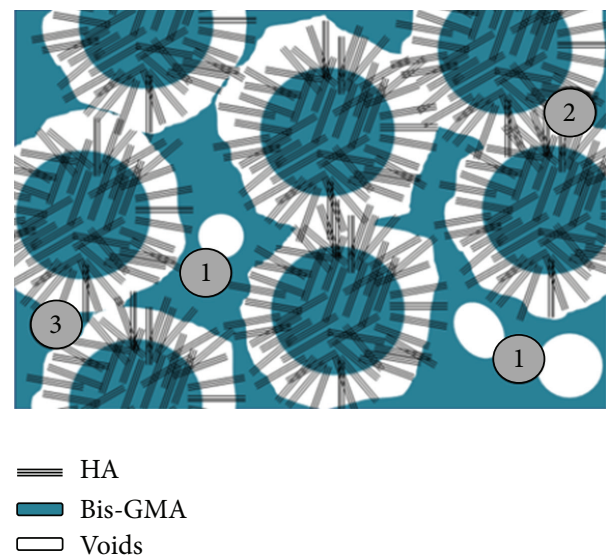

FIgure 7: Three-dimensional structure of the HA-Bis-GMA composite.
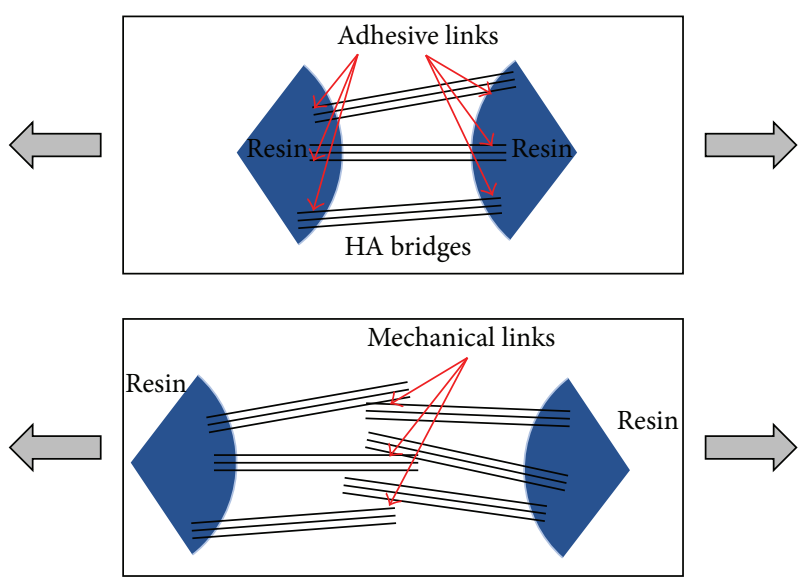

FIGURE 8: The mechanisms of transfer of the stresses between micelles.

distribution obstacles the stress transfer at the resin/filler interface. Thus, low mechanical performances are observed.

The mechanisms of transfer of the stresses in 2 and 3 zones could be mainly two (Figure 8): the first can be called adhesive links and it is characterized by the formation of bridges of HA between two neighboring micelles. The second is named as mechanical link and it is mainly due to friction between the micelles that are in contact.

With regard to the stiffening of the composite, adhesive links are better than the mechanical links. In the former, bridges of HA resist better to the tensile stress applied despite the mechanical links (this structural configuration is characterized by a low mechanical resistance to shear).

For the sake of comparison, we also tested some commercial composites, for which the mean values of flexural modulus and standard deviations (SD) are summarized in Table 5.

In Figure 9, the comparison between three commercial dental restorative materials and $20 \% \mathrm{HA} / \mathrm{Silica}$ samples is reported.
TABLE 5: Results of mechanical properties of four evaluated composites.

\begin{tabular}{lccc}
\hline Materials & $\begin{array}{c}\text { Young's } \\
\text { modulus } E \\
(\mathrm{MPa})^{\mathrm{a}}\end{array}$ & $\begin{array}{c}\text { Flexural } \\
\text { strength } \sigma \\
(\mathrm{MPa})^{\mathrm{a}}\end{array}$ & Strain $\varepsilon_{\max }$ \\
\hline Durafill VS & $2294(8.5)^{\mathrm{a}}$ & $54.37(5)^{\mathrm{a}}$ & 0.02069 \\
Renamel & $4000(10.9)$ & $80.29(11)$ & 0.01376 \\
Quadrant LC & $3800(9.2)$ & $70.15(3)$ & 0.01184 \\
\hline a Mean value (standard deviation) & &
\end{tabular}

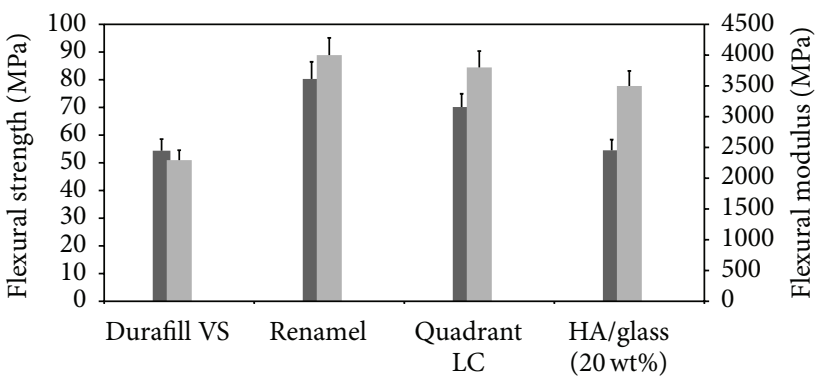

FIGURE 9: Flexural strength (dark grey) and elastic modulus (light grey) of four-resin based composite.

Renamel composite showed much higher mechanical performances, with a maximum flexural stress of $80.29 \mathrm{MPa}$. $20 \% \mathrm{HA} /$ Silica and Durafill VS samples showed no significant difference in flexural strength with a mean value that is around 54.5 MPa. Renamel and Quadrant LC showed no significant difference with a flexural strength, respectively, of $80 \mathrm{MPa}$ and $70 \mathrm{MPa}$.

ANOVA revealed that Renamel showed higher modulus (4000 MPa) which was statistically significant in comparison with Durafill VS $(P<0.05)$. Durafill had the lowest flexural modulus (2294 MPa), while between Renamel and Quadrant LC (3800 MPa) there was no significant difference; in addition, also Quadrant LC and 20\% HA/Silica composite $(3500 \mathrm{MPa})$ do not show any significant difference $(P>0.05)$.

Renamel composite outlines a stiff and brittle behavior which reflects the mean values detected for natural teeth. On the contrary, Durafill and 20\% HA/Silica do not show effective stiffness but possess good ductility which is useful in the presence of internal stresses or dynamic loads.

The SEM analysis of the HA-Bis-GMA composite has shown adhesion problems between filler and polymer matrix that it could be solved by using of surfactants or silane modified coupling agents. Their addiction allows improving the interaction between the constituents of the dental paste. The addition of plasticizers with low viscosity could also be used to improve the workability of the composite by reducing the number of voids. Such adhesion problems are not present in the commercial composite $[24,25]$.

3.1. Biocompatibility Tests. The biocompatibility of these innovative formulations with different rate of HA whiskers was assessed by evaluating the toxicity in an immortalized 


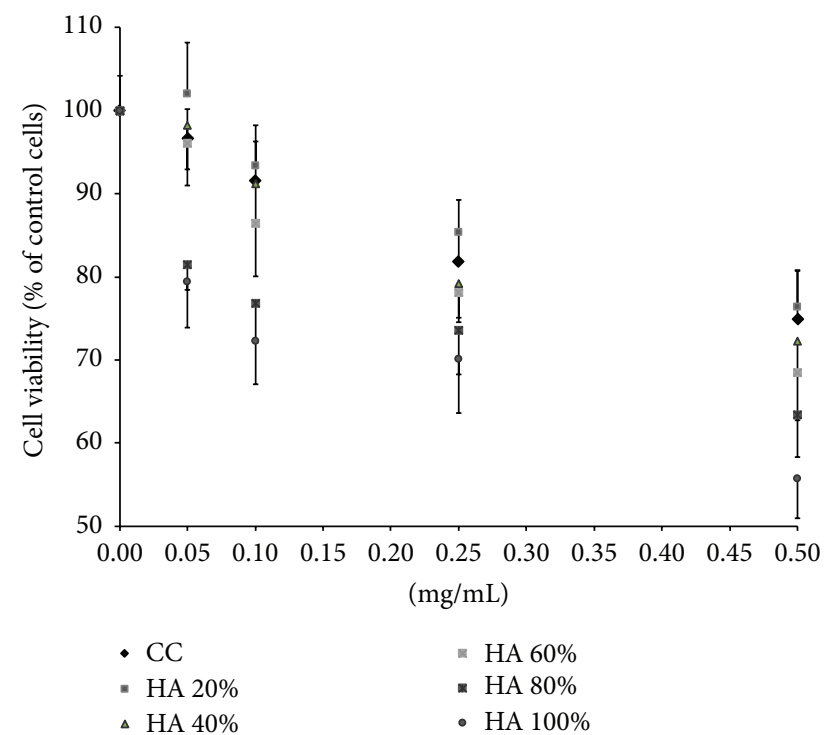

FIGURE 10: Effects of various composite powders modification (exchange of silica filer with HA whiskers \%) on THP-1 cell viability. THP-1 cells were treated with different concentrations of HA or CC $(0.05-0.5 \mathrm{mg} / \mathrm{ml})$ for $48 \mathrm{~h}$, and then cell viability was assessed by the MTT assay. Results are expressed as percentages of untreated cultures. Data are the mean \pm SEM from five separate experiments. Statistically significant differences were evaluated by one-way ANOVA, followed by Newman-Keuls post hoc test. ${ }^{*} p<$ $0.05,{ }^{* *} p<0.01$, and $p<0.001$ significant differences in comparison to untreated cells.

human monocyte THP-1 cell line. We also compared the effects of HA whiskers with commercial composite.

The incubation of THP-1 cells with different concentrations of various composite powders for $48 \mathrm{hs}$ caused a dose-dependent decrease in cell viability measured by MTT assay (Figure 10). In particular, the resin composites with highest HA content (80-100\%), at all concentrations, exerted cytotoxic effects on THP-1 cell culture, reducing cell viability up to $45 \%$ at the highest concentration in comparison to control cells $(P<0.001)$. Composites with $40-60 \%$ HA content were also cytotoxic. The cell viability was significantly reduced $(P<0.05)$ in a concentration range of $0.25-$ $0.5 \mathrm{mg} / \mathrm{ml}$, while the incubation with 0.05 and $0.1 \mathrm{mg} / \mathrm{ml}$ of HA $40-60 \%$ produced no significant reduction of cell viability, by $10-15 \%$ in comparison to untreated cells $(P>$ 0.05). This was mainly due to the shielding effect of the hydroxyapatite fillers that, at high content, reduce the degree of conversion of the matrix, consequently increasing the availability of cytotoxic uncured monomers and initiator [21].

Among all HA composites, HA-20 demonstrated very low cytotoxic effects. In agreement with other results [26], it is possible to speculate that slight but no significant increase in MTT values occurring in the presence of HA-20 may be ascribed to a partial effect on cell proliferation.

After incubation of THP-1 cells with HA $20 \%$ at concentrations ranging between 0.05 and $0.25 \mathrm{mg} / \mathrm{ml}$, we did not observe significant changes in cell viability in comparison with control cells. Similarly, commercial composite in the same concentration range was not cytotoxic. However, at highest concentration $(0.05 \mathrm{mg} / \mathrm{ml})$ both $\mathrm{HA} 20 \%$ and commercial compounds showed a significant increase in cell death, reducing cell viability by $25 \%$ in comparison to untreated cells $(P<0.05)$.

\section{Conclusion}

The study on a new hybrid composite, conducted thus far, shows how mechanical properties can be compared with that of commercial composites by the addition of a $20 \%$ volume ratio of hydroxyapatite with whisker morphology on conventional silica filler. Until this range, the links between micelles provide a good interaction at matrix/filler interface. Biocompatibility assessment confirms the better response of THP-1 cell culture to $20 \%$ HA based composite in comparison with both commercial composites to higher wHA concentrations, confirming promising results achieved by the replacement of silica filler with small amount of hydroxyapatite (up to $20 \%)$. The effect of adding hydroxyapatite whiskers filler on the bond strength with dentine must be further investigated.

\section{Conflict of Interests}

The authors declare that there is no conflict of interests regarding the publication of this paper.

\section{References}

[1] F. Liu, R. Wang, Y. Cheng, X. Jiang, Q. Zhang, and M. Zhu, "Polymer grafted hydroxyapatite whisker as a filler for dental composite resin with enhanced physical and mechanical properties," Materials Science and Engineering C, vol. 33, no. 8, pp. 4994-5000, 2013.

[2] N. Kantharia, S. Naik, S. Apte, M. Kheur, S. Kheur, and B. Kale, "Nano-hydroxyapatite and its contemporary applications," Journal of Dental Research and Scientific Development, vol. 1, pp. 1519, 2014.

[3] C. Domingo, R. W. Arcìs, A. López-Macipe et al., "Dental composites reinforced with hydroxyapatite: mechanical behavior and absorption/elution characteristics," Journal of Biomedical Materials Research, vol. 56, no. 2, pp. 297-305, 2001.

[4] H. Zhang and B. W. Darvell, "Mechanical properties of hydroxyapatite whisker-reinforced bis-GMA-based resin composites," Dental Materials, vol. 28, no. 8, pp. 824-830, 2012.

[5] Y.-R. Zhang, W. Du, X.-D. Zhou, and H.-Y. Yu, "Review of research on the mechanical properties of the human tooth," International Journal of Oral Science, vol. 6, no. 2, pp. 61-69, 2014.

[6] J. M. Corrêa, M. Mori, H. L. Sanches, A. D. da Cruz, E. Poiate Jr., and I. A. V. Pola Poiate, "Silver nanoparticles in dental biomaterials," International Journal of Biomaterials, vol. 2015, Article ID 485275, 9 pages, 2015.

[7] C. Domingo, R. W. Arcìs, E. Osorio et al., "Hydrolytic stability of experimental hydroxyapatite-filled dental composite materials," Dental Materials, vol. 19, no. 6, pp. 478-486, 2003.

[8] H. Zhang and B. W. Darvell, "Failure and behavior in water of hydroxyapatite whisker-reinforced bis-GMA-based resin composites," Journal of the Mechanical Behavior of Biomedical Materials, vol. 10, pp. 39-47, 2012. 
[9] C. Santos, R. L. Clarke, M. Braden, F. Guitian, and K. W. M. Davy, "Water absorption characteristics of dental composites incorporating hydroxyapatite filler," Biomaterials, vol. 23, no. 8, pp. 1897-1904, 2002.

[10] M. Lezaja, D. N. Veljovic, B. M. Jokic, I. Cvijovic-Alagic, M. M. Zrilic, and V. Miletic, "Effect of hydroxyapatite spheres, whiskers, and nanoparticles on mechanical properties of a model BisGMA/TEGDMA composite initially and after storage," Journal of Biomedical Materials Research Part B: Applied Biomaterials, vol. 101, no. 8, pp. 1469-1476, 2013.

[11] R. W. Arcís, A. López-Macipe, M. Toledano et al., "Mechanical properties of visible light-cured resins reinforced with hydroxyapatite for dental restoration," Dental Materials, vol. 18, no. 1, pp. 49-57, 2002.

[12] A. Tunçel, A. K. Özdemir, Z. Sümer, F. Hürmüzlü, and Z. Polat, "Cytotoxicity evaluation of two different composites with/without fibers and one nanohybrid composite," Dental Materials Journal, vol. 25, no. 2, pp. 267-271, 2006.

[13] H. Yilmaz, C. Aydin, A. Çaglar, and A. Yaşar, "The effect of glass fiber reinforcement on the residual monomer content of two denture base resins," Quintessence International, vol. 34, no. 2, pp. 148-153, 2003.

[14] V. Stanić, S. Dimitrijević, J. Antić-Stanković et al., "Synthesis, characterization and antimicrobial activity of copper and zincdoped hydroxyapatite nanopowders," Applied Surface Science, vol. 256, no. 20, pp. 6083-6089, 2010.

[15] A. U. J. Yap and S. H. Teoh, "Comparison of flexural properties of composite restoratives using the ISO and mini-flexural tests," Journal of Oral Rehabilitation, vol. 30, no. 2, pp. 171-177, 2003.

[16] L. M. Bonaccorsi, C. Borsellino, L. Calabrese et al., "Performances evaluation of a Bis-GMA resin-based composite for dental restoration," Acta Medica Mediterranea, vol. 28, no. 2, pp. 163-166, 2012.

[17] V. Mittal, Nanocomposites with Biodegradable Polymers: Synthesis, Properties, and Future Perspectives, Oxford University Press, Oxford, UK, 2011.

[18] A. Yasukawa, S. Ruike, K. Gotoh, and K. Kandori, "Ultraviolet shielding properties of cotton fabric supported by cerium-calcium hydroxyapatite solid solution particles," Textile Research Journal, vol. 84, no. 15, pp. 1578-1585, 2014.

[19] A. Apicella, M. Simeone, R. Aversa, A. Lanza, and D. Apicella, "Light shielding effect of overlaying resin composite on the photopolymerization cure kinetics of a resin composite and a dentin adhesive," Dental Materials, vol. 21, no. 10, pp. 954-961, 2005.

[20] J. R. David, O. M. Gomes, J. C. Gomes, A. D. Loguercio, and A. Reis, "Effect of exposure time on curing efficiency of polymerizing units equipped with light-emitting diodes," Journal of Oral Science, vol. 49, no. 1, pp. 19-24, 2007.

[21] V. C. B. Leitune, F. M. Collares, R. M. Trommer, D. G. Andrioli, C. P. Bergmann, and S. M. W. Samuel, "The addition of nanostructured hydroxyapatite to an experimental adhesive resin," Journal of Dentistry, vol. 41, no. 4, pp. 321-327, 2013.

[22] K. L. Van Landuyt, J. Snauwaert, J. De Munck et al., "Systematic review of the chemical composition of contemporary dental adhesives," Biomaterials, vol. 28, no. 26, pp. 3757-3785, 2007.

[23] S. Kalachandra, "Influence of fillers on the water sorption of composites," Dental Materials, vol. 5, no. 4, pp. 283-288, 1989.

[24] F. Fabiano, C. Borsellino, L. M. Bonaccorsi, L. Calabrese, V. Fabiano, and G. Mavilia, "Influence of irradiation exposure time on the depth cure of restorative resin composite," Atti della
Accademia Peloritana dei Pericolanti-Classe di Scienze Fisiche, Matematiche e Naturali, vol. 92, supplement 1, pp. A1-A8, 2014.

[25] L. Calabrese, F. Fabiano, L. M. Bonaccorsi, V. Fabiano, and C. Borsellino, "Evaluation of the clinical impact of ISO 4049 in comparison with miniflexural test on mechanical performances of resin based composite," International Journal of Biomaterials, vol. 2015, Article ID 149798, 7 pages, 2015.

[26] L. Hernández, J. Parra, B. Vázquez et al., "Injectable acrylic bone cements for vertebroplasty based on a radiopaque hydroxyapatite. Bioactivity and biocompatibility," Journal of Biomedical Materials Research Part B: Applied Biomaterials, vol. 88, no. 1, pp. 103-114, 2009. 

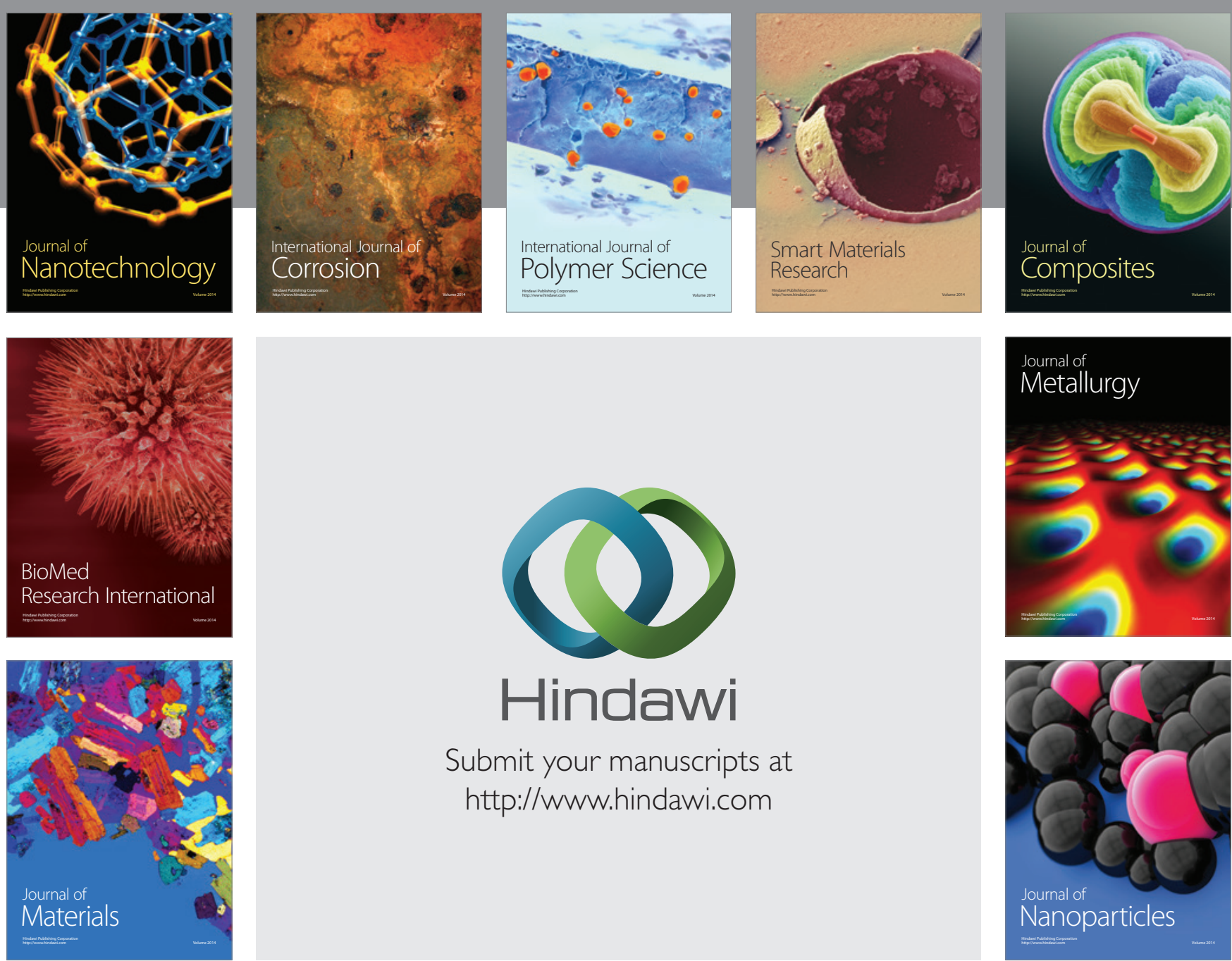

\section{Hindawi}

Submit your manuscripts at

http://www.hindawi.com

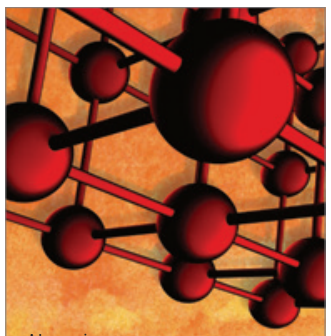

Materials Science and Engineering
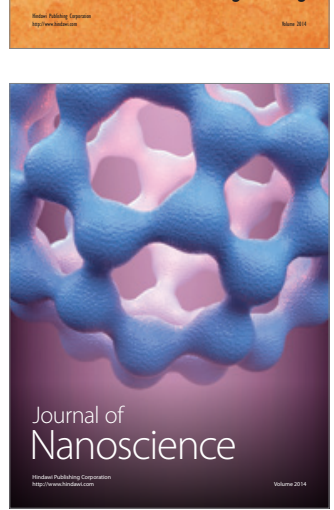
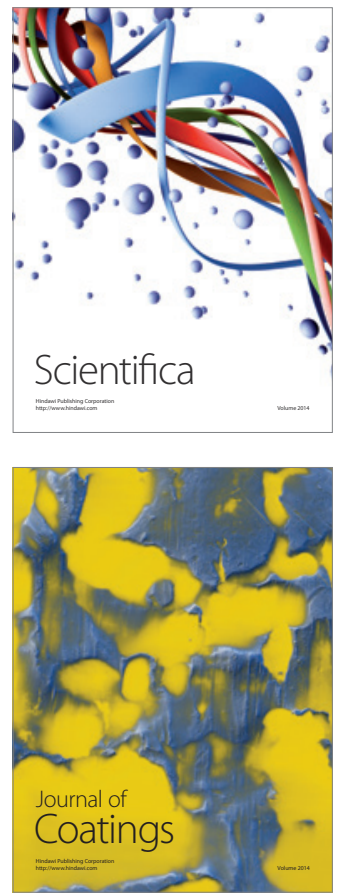
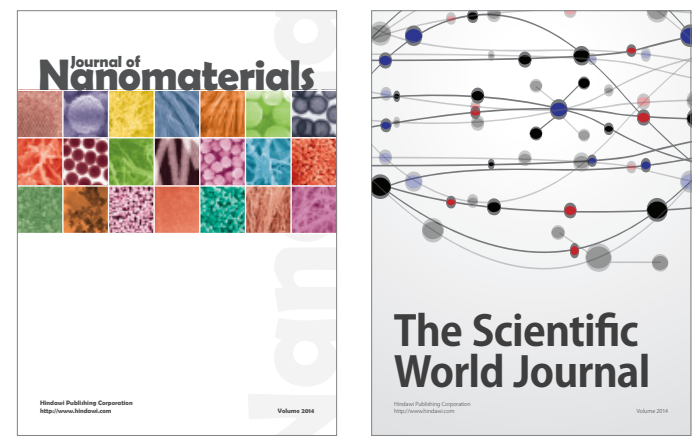

The Scientific World Journal
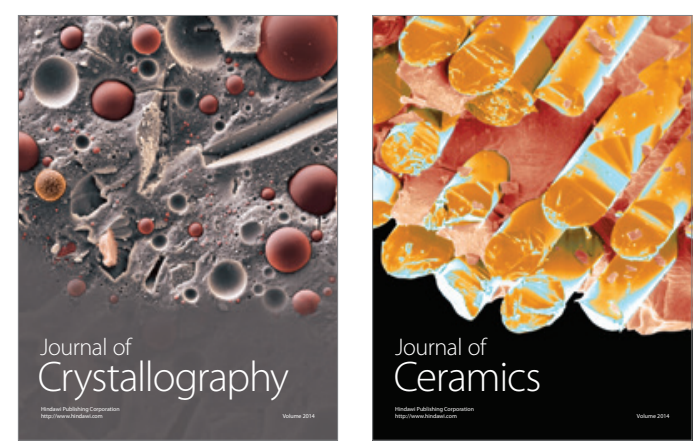
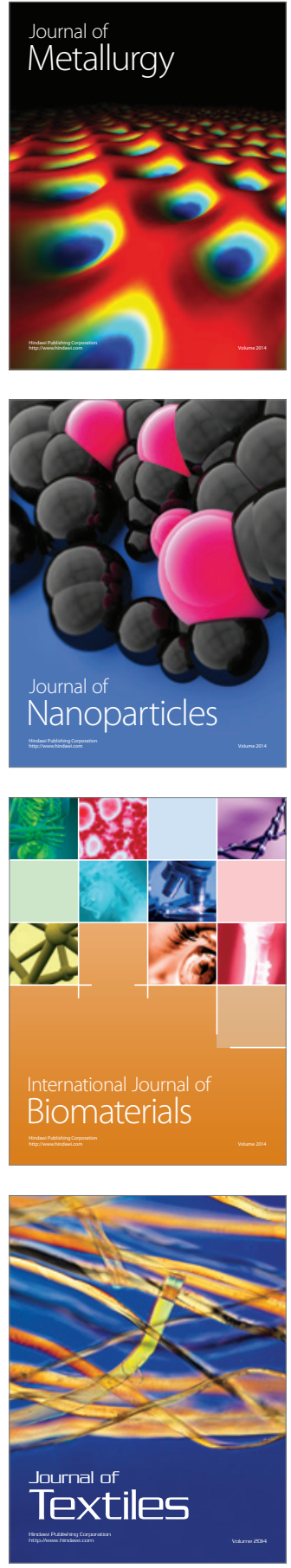